\title{
Evaluación poblacional de Dipteryx micrantha en la cuenca del río Las Piedras, Madre de Dios (Perú)
}

\author{
Population evaluation of Dipteryx micrantha in the \\ Las Piedras river basin, Madre de Dios (Peru)
}

Tatiana Espinosa ${ }^{1,{ }^{*}}$ y Daniel Valle ${ }^{2}$

Recibido: 28 agosto 2020 | Aceptado: 08 diciembre 2020 | Publicado en línea: 18 diciembre 2020 Citación: Espinosa, T; Valle, D. 2020. Evaluación poblacional de Dipteryx micrantha en la cuenca del río Las Piedras, Madre de Dios (Perú). Revista Forestal del Perú 35 (3, Número Especial): 7685. DOI: http://dx.doi.org/10.21704/rfp.v35i3.1603

\begin{abstract}
Resumen
El Shihuahuaco (Dipteryx spp.) es una de las especies emergentes conspicuas de los bosques amazónicos. Actualmente, su futuro está amenazado por la sobre-extracción comercial y aparente baja tasa de regeneración. Esta investigación tiene por objetivo gestionar información primaria de una población de Shihuahuaco (Dipteryx micrantha) en un área boscosa en la cuenca del río Las Piedras - Madre de Dios, con la finalidad de evaluar su viabilidad en el tiempo. El área de estudio forma parte de una concesión forestal, que antiguamente estuvo sujeta a la tala selectiva de especies maderables valiosas cómo el cedro (Cedrela odorata) y caoba (Swietenia macrophylla). Utilizando métodos mixtos relacionados con el inventario poblacional y el cálculo alométrico de la edad se determinó la capacidad de regeneración, reclutamiento, estructura de edades y clases diamétricas para Dipteryx micrantha. El estudio se llevó a cabo en un área de 155 ha para el muestreo de individuos juveniles y adultos. Dentro de esta área se realizaron los muestreos de latizales, regeneración natural y plantones. Se estimó que existen $275 \mathrm{ind} / \mathrm{ha}$ de regeneración natural, 0,91 ind/ha de plantones, cero latizales, 0,06 ind/ha juveniles, y 0,71 ind/ha de árboles adultos. El promedio de DAP fue de $87,66 \mathrm{~cm}(\mathrm{SDE}+/-2.99 \mathrm{~cm})$, y las clases de diamétricas presentaron una distribución normal, por lo que esta población de Dipteryx micrantha es representativa de toda la concesión. El DAP promedio ha permitido estimar la edad promedio de estos árboles en 684.83 años (SDE +/- 29,50). La principal conclusión de esta investigación es que los árboles de Dipteryx micrantha de esta área son muy antiguos, y que su regeneración y reclutamiento es bajo. Asimismo, no sería sostenible ninguna forma de extracción con fines maderables.
\end{abstract}

Palabras clave: Shihuahuaco, estado de conservación, bosque amazónico, cumarú, regeneración natural

${ }^{1}$ Arbio Perú. Asociación para la Resiliencia del Bosque frente a la Interoceánica.

${ }^{2}$ Universidad Científica del Sur. Carrera de Turismo Sostenible y Hotelería.

* Autor de Correspondencia: tatiana@arbioperu.org 


\begin{abstract}
The Shihuahuaco (Dipteryx spp.) is one of the most conspicuous emerging species in Amazon forests. Currently, its future is threatened by commercial over-extraction, which is complicated by its apparent low regeneration rate. The objective of this research is to manage primary information on a population of Shihuahuaco (Dipteryx micrantha) in a wooded area in the Las Piedras - Madre de Dios river basin, in order to evaluate its diameter structure, and thereby infer its viability and sustainability over time. The study area is part of a forest concession, which was formerly subject to logging of valuable timber species such as cedar (Cedrela odorata) and big leaf mahogany (Swietenia macrophylla). Using mixed methods related to the population inventory and the allometric calculation of age, the regeneration capacity, recruitment, age structure and diameter classes for Dipteryx micrantha were determined. The study was carried out in an area of 155 ha for the sampling of juvenile and adult individuals. Within this area the sampling of latizales, natural regeneration and seedlings were carried out. It was estimated that there are $275 \mathrm{ind} / \mathrm{ha}$ of natural regeneration, 0.91 ind/ha of seedlings, zero latizales, $0.06 \mathrm{ind} /$ ha juveniles, and $0.71 \mathrm{ind} / \mathrm{ha}$ of adult trees. The mean $\mathrm{DBH}$ was $87.66 \mathrm{~cm}(\mathrm{SDE}+/-2.99 \mathrm{~cm})$, and the diameter classes presented a normal distribution, so this population of Dipteryx micrantha is representative of the entire concession. The average DBH has made it possible to estimate the average age of these trees at 684.83 years (SDE +/-29.50). The main conclusion of this investigation is that the Dipteryx micrantha trees in this area are so old, and that their regeneration and recruitment is low. Likewise, any form of extraction for timber purposes would not be sustainable.
\end{abstract}

Palabras clave: Shihuahuaco, conservation status, Amazon forest, cumarú, natural regeneration

\section{Introducción}

Los bosques húmedos en la Amazonía peruana son complejos y heterogéneos, y en especial los bosques amazónicos de tierras bajas inundables, que son ecosistemas que contienen una considerable diversidad de especies arbóreas (OSINFOR 2013). Estos bosques tienen una composición florística muy compleja o altamente heterogénea, que se ha estimado en más de 6727 especies diferentes de árboles (Cardoso et al. 2017). Actualmente, la autoridad nacional forestal y de fauna silvestre de Perú - SERFOR, ha identificado 275 especies arbóreas con fines maderables (RDE n¹18-2019-MINAGRI-SERFOR-DE). De este universo de especies forestales, el comercio nacional de maderas se limita a 120 especies maderables, de las cuales solo 20 han sido debidamente estudiadas e identificadas, por lo que un elevado porcentaje son consideradas como maderas corrientes (OSINFOR 2013).

La creciente demanda de madera para cubrir los mercados nacionales e internacionales ha ocasionado que se incremente la presión de la tala sobre las pocas especies consideradas de valor comercial (Putzel 2010), pero las actividades y alteraciones de origen antropogénico pueden impactar la estructura del bosque, así como su funcionamiento y composición (Oliveira et al. 2007). En los bosques amazónicos fragmentados, la tasa de mortalidad de los grandes árboles se incrementa debido a una baja en su fecundidad. Con esto, se disminuye la estructura y complejidad del bosque, alterando los ciclos biogeoquímicos, la evapotranspiración, ciclo de carbono, entre otros (Laurance et al. 2000).

Putzel (2010) mostró que en el año 2006 las declaraciones ante la SUNAT de los cargamentos de madera aserrada ascendían a 56425 $\mathrm{m}^{3}$. De este volumen total de madera, el 63\% era embarcado a China, y de este volumen de madera exportada, el 95\% corresponde a especies de tres géneros de árboles de madera dura varias especies del género Dipteryx spp. y las especies Myroxylon balsamum (L.) Harms, y Manilkara bidentata (A. DC.) A. Chev. Además de tener un valor económico alto, las especies de estos géneros demuestran una importancia alta para los procesos ecológicos del bosque (Putzel 

del río Las Piedras, Madre de Dios (Perú)

et al. 2008), aunque no son categorizados bajo amenaza. De los tres, la alta extracción de Dipteryx spp. es sumamente preocupante por las observaciones que cada año hay menos árboles en el bosque.

De acuerdo con la Lista oficial de Especies Forestales del Perú (RDE nº118-2019-MINAGRI-SERFOR-DE), el árbol conocido como Shihuahuaco corresponde a dos especies Dipteryx charapilla (J.F. Macbr.) Ducke y Dipteryx micrantha Harms. Sin embargo, Aldana et al. (2016) lograron diferenciar tres grupos de especies del género Dipteryx en los bosques naturales de la Amazonía peruana. Según Aldana et al. (2016), estos grupos presentan características morfológicas particulares y distribución geográfica distintos. De acuerdo con Díaz (2018), las poblaciones de Dipteryx micrantha presentan una gran semejanza genética entre ellas, aunque también existe una diferenciación de entre las poblaciones ubicadas en Madre de Dios (Manu e Iñapari) respecto al resto de localidades.

Mientras que recientes estudios reconocen por los menos tres taxa, la autoridad forestal estatal reconoce solo $D$. micrantha y $D$. charapilla (RDE n¹18-2019-MINAGRI-SERFOR-DE). Por este motivo, la determinación de especies de uso forestal es crucial, dado que, en términos económicos, la identificación taxonómica inadecuada puede conducir a una sobreestimación de la abundancia de algunas especies, ya que consideraría la abundancia de dos taxones diferentes como uno y los pondría en riesgo de sobreexplotación (Garcia-Davila et al. 2019).

En este sentido, Garcia-Davila et al. (2019) consideran que la determinación de las especies de Shihuahuaco debe reorientar el establecimiento de cuotas de explotación diferenciadas de acuerdo con la especie y su densidad de población de manera que permita el uso sostenible del bosque centrado en las especies abundantes y ampliamente distribuidas.

Un aspecto necesario para considerar en el aprovechamiento forestal sostenible es la determinación de las edades de la población de Shihuahuaco. En este sentido, científicos como Chambers et al. (1998) integraron el cálculo para estimar edades en árboles tropicales en base a anillos de crecimiento con la datación de Carbono 14 en los troncos. Así, Jenkins (2009) estudió la relación entre la formación de anillos de crecimiento anual y acumulación del isotopo carbono 14 (14C) en especímenes de Dipteryx micrantha colectados en Madre de Dios, determinando la tasa de envejecimiento anual del árbol en relación con la formación de anillos de crecimiento. Jenkins (2009) encontró que en los primeros 50 años de vida, $D$. $m i$ crantha crece entre un rango de 2 a $5 \mathrm{~mm} / \mathrm{año}$, con un promedio de 3 a 3,5 mm/año, y luego de los 200 años sólo crecían un promedio de $1 \mathrm{~mm} / \mathrm{año.}$

Para Chambers et al. (1998), las edades de los árboles de las selvas tropicales proporcionan información crítica para comprender su dinámica poblacional, determinar los patrones históricos de los ciclos de perturbación, así como para desarrollar prácticas forestales sostenibles y calcular las tasas de ciclo del carbono. Sin embargo, la historia de vida ecológica de la mayoría de estos árboles tropicales es aún desconocida. Incluso, las edades de los grandes árboles del bosque aún no han sido determinadas. Para este estudio, se consideró realizar una evaluación poblacional de la especie Dipteryx micrantha, con la finalidad de evaluar su estado $y$ viabilidad poblacional a futuro en la cuenca del río Las Piedras, provincia Tambopata, departamento Madre de Dios. Específicamente, se buscó determinar estructura diamétrica y determinación de las edades cronológicas de los árboles evaluados.

\section{Materiales y Métodos}

\section{Área de estudio}

Arbio-Perú administra la concesión forestal con fines de conservación que está ubicada en la parte media cuenca del río Las Piedras, distrito de Las Piedras, departamento de Madre de Dios; y se ubica a $48,75 \mathrm{~km}$ al nornoroeste de Puerto Maldonado.

En los últimos 30 años, los bosques que actualmente son parte de esta concesión forestal de Arbio-Perú fueron sometidos a la tala selectiva de especies valiosas como la caoba (Swie- 
tenia macrophylla King) y el cedro (Cedrela odorata L.), quedando en el área especies de bajo valor comercial.

De acuerdo con el mapa climático del Perú elaborado por SENAMHI (2020), la zona de estudio mantiene la caracterización climática de Puerto Maldonado, con una temperatura media histórica entre $32,2^{\circ} \mathrm{C}$ (setiembre) a $16,6^{\circ} \mathrm{C}$ (julio), y con una precipitación acumulada promedio de $2062,6 \mathrm{~mm}$. Esta es una selva muy lluviosa y calidad clasificada como Selva Tropical Muy Húmeda - A(r) A' H4. La estación biológica de Arbio-Perú, que se ubica dentro de la concesión, está a una altitud de $215 \mathrm{msnm}$. El terreno en la concesión es ligeramente ondulado con una altitud máxima de $235 \mathrm{msnm}$.

De acuerdo con lo indicado en el mapa de ecosistemas del Perú (MINAM 2019), la concesión gestionada por Arbio-Perú le corresponde los ecosistemas de Bosque Aluvial Inundable (B-ai) y el Bosque de Colina Baja (Bcb). Los suelos están sometidos a inundación temporal (semanas o pocos meses) o casi permanente; el bosque con sotobosque ralo o abierto puede presentar 3 o 4 estratos con un dosel o cúpula de árboles que alcanzan entre 20 a 25 metros de alto e individuos emergentes de hasta 30 metros de altura (MINAM 2019).

\section{Evaluación en campo}

Para la evaluación de individuos de $D$. micrantha, se realizaron tres expediciones entre los meses de abril a setiembre 2019. Se identificaron árboles de $D$. micrantha en toda su gradiente de tamaño, clasificándolos de acuerdo con las categorías propuestas por Romo et al. (2004) y MINAGRI (2013) en cinco clases de tamaño diferentes: regeneración natural $(<1$ $\mathrm{cm}$ de diámetro del tallo), plantones (diámetro del tallo $\geq 1 \mathrm{~cm} \mathrm{y}<4 \mathrm{~cm}$ ), latizales (diámetro a la altura del pecho, DAP $\geq 4 \mathrm{~cm} \mathrm{y}<10 \mathrm{~cm}$ ), fustales o juveniles (DAP $\geq 10 \mathrm{~cm}$ y $<40 \mathrm{~cm}$ ), y árboles adultos (DAP $\geq 40 \mathrm{~cm})$.

Para la medición y registro de los árboles se usó la red de caminos y vías de la concesión, que en total tienen $15,5 \mathrm{~km}$. Estos caminos se usaron como transectos para la evaluación.
Los latizales se evaluaron a ambos lados de los transectos, hasta una distancia de 5 metros por lado. Para la evaluación de árboles adultos y juveniles se realizaron los registros en transectos. Sin embargo, a diferencia de la evaluación de los latizales, los árboles adultos evaluados fueron individuos ubicados hasta 50 metros a cada lado de los transectos, lo que representa un total de 155 hectáreas evaluadas.

Para la evaluación de regeneración natural (RN) y plantones se determinaron cuatro parcelas partiendo de un árbol madre hacia los puntos cardinales, completando un cuadrado de $10 \mathrm{~m}$ de lado con el árbol en la zona central. En estas parcelas realizamos un muestreo para la evaluación de RN y de plantones. La elección de árboles a evaluar siguió dos criterios i) Conteo de uno sí, dos no (captados visualmente en la trocha "Shihuahuaco"), es decir, por cada árbol evaluado en el transecto se dejó sin evaluar los dos árboles siguientes; ii) En aplicación al primer criterio, si se evidenciaba que algún árbol caía cerca o que puede entrecruzarse con las parcelas de otro árbol, entonces era descartado y se procedía pasar al siguiente árbol adulto. Luego, se ubicaron parcelas rectangulares de 50 por $10 \mathrm{~m}$ con orientación Norte, Sur, Este Oeste, siendo cuatro parcelas por árbol evaluado. A este arreglo de parcelas, los llamamos "parcelas centrales" y "parcelas rectangulares", siendo en total 8 parcelas por cada árbol evaluado (Figura 1$)$.

Se evaluaron estos dos tipos de parcelas para obtener información sobre la existencia de diferencias significativas entre la presencia de $\mathrm{RN}$ y plantones muy cerca del árbol madre o en un gradiente de alejamiento de hasta 50 metros del árbol madre (debido a que la semilla de Dipteryx micrantha es pesada y generalmente cae cerca del árbol madre). A cada árbol adulto y juvenil se le colocó una etiqueta con un código único para poder realizar una reevaluación en el futuro.

Las mediciones de los individuos adultos incluyeron la circunferencia y estimación de la altura con un Hipsómetro digital Nikon Forestry Pro. Los datos de la medición fueron ingresados al aplicativo Censa Árbol 1.2. Solo 


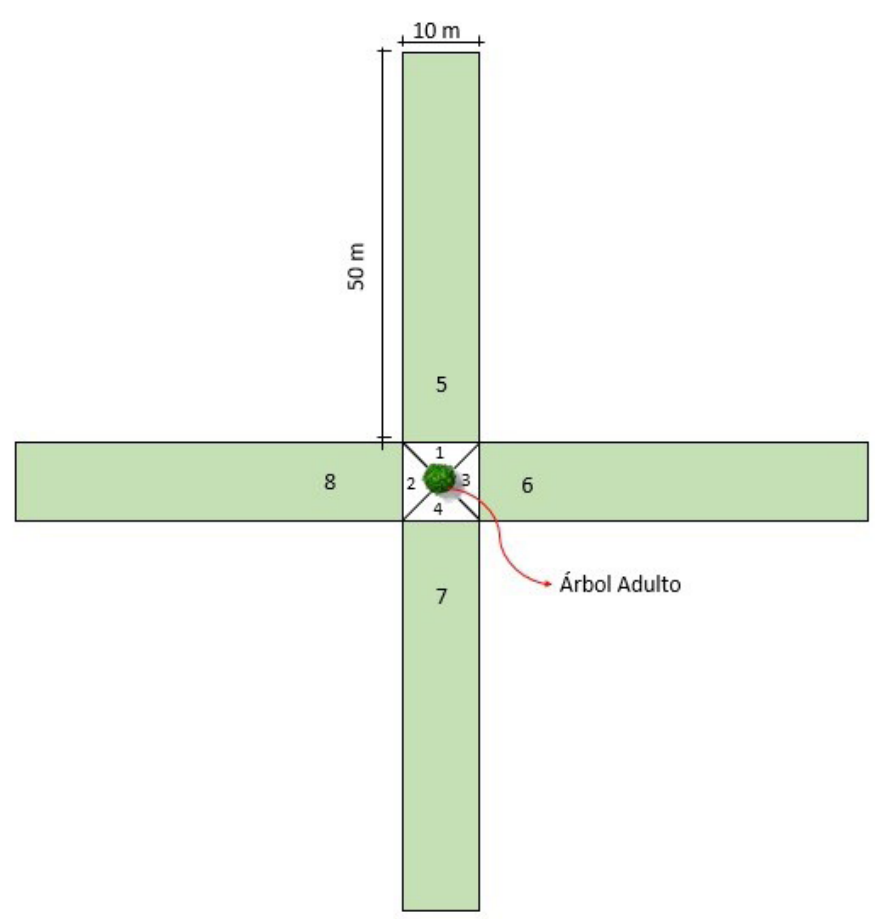

Figura 1. Diagrama de parcelas de evaluación de Regeneración Natural en la concesión Arbio Perú.

para los árboles de aletas grandes, se hizo una proyección del diámetro usando una vara, ubicada en horizontal a la altura del pecho.

Las variables registradas para árboles adultos fueron la posición de cada árbol, expresada en coordenadas UTM con ayuda de una Tablet Galaxy Tab S2 y GPS externo GlobalSat BT821 Bluetooth GPS Receiver, diámetro a la altura del pecho (DAP), altura en metros. Luego, del total de árboles registrados, se seleccionaron 12 individuos para realizar la evaluación de la regeneración natural. Para toma de datos de campo se usó Censa Arbol 1.2, aplicación basada en tecnología ESRI Survey 123 para censo de árboles

\section{Análisis de datos}

Para el análisis estadístico en la determinación de las clases de edades diamétricas se usó el aplicativo PAST3.18 (Hammer et al. 2001). Este análisis incluyó la prueba de normalidad Shapiro-Wilks.
Para la estimación de las edades cronológicas de los árboles de D. micrantha se usaron los resultados del estudio realizado por Jenkins (2009), pudiendo proyectar la siguiente formula alométrica para la estimación de edades:

$$
\text { Edad }=-181.651+(0.988)^{\star} \text { DAP }
$$

Donde DAP = Diámetro a la Altura del Pecho que se expresa en $\mathrm{mm}$.

\section{Resultados}

\section{Estructura de clases diamétricas}

En total se evaluaron 120 árboles (DAP $\geq 10$ $\mathrm{cm})$. Se clasificaron en siete clases diamétricas de $20 \mathrm{~cm}$ cada una (Figura 2). Del análisis de las clases diamétricas, se registró que el 71,93\% de árboles tiene diámetros mayores a $70 \mathrm{~cm}$, obteniéndose un promedio poblacional estimado de 87,66 cm (Std +/- 2,993 cm). Además, el 38,6\% de los individuos tienen DAP igual o mayor a $100 \mathrm{~cm}$. La distribución de las clases 


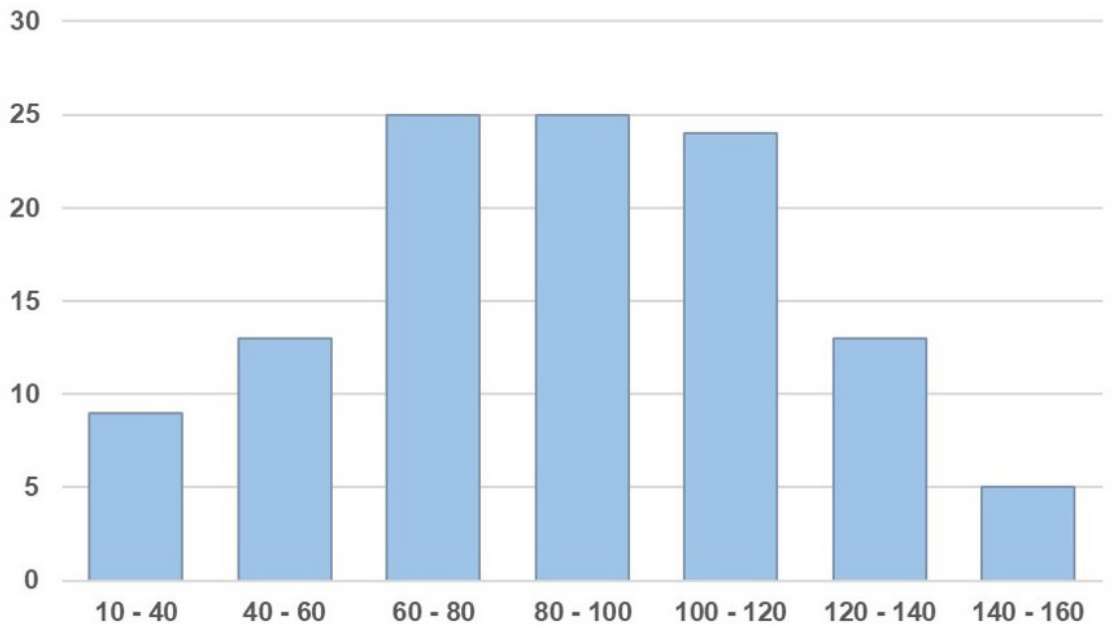

Figura 2. Diagrama de clases diamétricas de Dipteryx micrantha en la concesión Arbio Perú.

diamétricas presentó una distribución normal (el valor de la prueba de normalidad ShapiroWilks fue 0,947 $(p=0,05))$.

\section{Estimación de edades en D. micrantha}

En aplicación de la fórmula alométrica proyectada del estudio de Jenkins (2009), la edad promedio estimada de Dipteryx micrantha en el área de estudio fue de 684,83 años (Std $+/-29,5)$. Lo importante de este análisis es que el $87,5 \%$ de los árboles adultos evaluados tendrían más de 300 años, 68,33\% tendría más de 500 años, y el 15,83\% de los árboles evaluados tendrían más de 1000 años. La edad estimada del individuo más viejo sería de 1 327,9 años $(\mathrm{DAP}=152 \mathrm{~cm})$.

\section{Regeneración Natural y Plantones}

La evaluación de la regeneración natural (RN) se realizó en 12 puntos de muestreo, cada uno correspondiente a árboles adultos. En todo el muestro se encontró un total de 605 plantas de RN, y dos plantones de Shihuahuaco, uno con $88 \mathrm{~cm}$ de altura y $1,3 \mathrm{~cm}$ de diámetro, y el segundo con una altura de $160 \mathrm{~cm}$, y $1,5 \mathrm{~cm}$ de diámetro. En relación con la evaluación de RN, todas las plantas encontradas registraron alturas por debajo de los $50 \mathrm{~cm}$, con un promedio de $30 \mathrm{~cm}$.
El resultado estimado de $\mathrm{RN}$ es de 274 ind/ ha. Los plantones dieron una densidad 0,9 ind/ ha (Cuadro 1). La distribución de la $\mathrm{RN}$ no fue regular, porque un solo árbol entre los 120 evaluados presentó 496 plantas de RN, es decir, el $82 \%$ de las plantas de RN registradas estuvieron concentradas en un árbol madre.

\section{Latizales, árboles juveniles y adultos}

Durante la evaluación no se encontraron individuos dentro de la categoría de latizales. Con relación a los individuos juveniles, se contaron 10 individuos, los que representan el $8,3 \%$ de los individuos encontrados y equivale a 0,07 ind/ha. La cantidad de árboles adultos fue de 110 individuos, lo cual equivale a 0,71 ind/ha. (Cuadro 1).

\section{Discusión}

En base a recientes estudios realizados sobre el género Dipteryx en el Perú (Aldana et al. 2016; Garcia-Davila et al. 2019), ahora se posee evidencia suficiente para indicar que lo que conocemos como Shihuahuaco se trataría de tres entidades: $D$. charapilla (distribuido en Loreto), D. micrantha "morfotipo 1" (distribuido en Loreto y Ucuyali) y D. micrantha "morfotipo 2" (distribuido en Ucayali y Madre de Dios). Asimismo, Honorio et al. (2020) usando 

del río Las Piedras, Madre de Dios (Perú)

\begin{tabular}{|c|c|c|c|c|c|c|}
\hline Tipo & Característica & $\begin{array}{l}\text { Método de } \\
\text { muestreo }\end{array}$ & $\begin{array}{c}\text { Área } \\
\text { evaluada } \\
\text { (ha) }\end{array}$ & $\begin{array}{l}\text { Número de } \\
\text { individuos }\end{array}$ & $\begin{array}{c}\text { Densidad } \\
\text { Ind/ha }\end{array}$ & $\begin{array}{c}\text { Densidad } \\
\text { Ind/ha PN } \\
\text { Manu } \\
\text { (Romo 2004) }\end{array}$ \\
\hline $\begin{array}{l}\text { Regeneración } \\
\text { Natural }\end{array}$ & $\begin{array}{l}<1 \mathrm{~cm} \text { de diá- } \\
\text { metro del tallo }\end{array}$ & $\begin{array}{l}\text { Parcelas centra- } \\
\text { les y rectangu- } \\
\text { lares en árboles } \\
\text { adultos }\end{array}$ & 2.2 & 605 & 275 & $1-167$ \\
\hline Plantones & $\begin{array}{c}\text { Diámetro del } \\
\text { tallo } \geq 1 \mathrm{~cm} \mathrm{y}< \\
4 \mathrm{~cm}\end{array}$ & $\begin{array}{l}\text { Parcelas centra- } \\
\text { les y rectangu- } \\
\text { lares en árboles } \\
\text { adultos }\end{array}$ & 2.2 & 2 & 0.91 & $1.42-12.82$ \\
\hline Latizales & $\begin{aligned} \mathrm{DAP} & \geq 4 \mathrm{~cm} y \\
& <10 \mathrm{~cm}\end{aligned}$ & $\begin{array}{l}\text { A lo largo de } \\
\text { transectos (5 m } \\
\text { a cada lado) }\end{array}$ & 15.5 & 0 & 0 & 0.018 \\
\hline $\begin{array}{l}\text { Fustales } \\
\text { (juveniles) }\end{array}$ & $\begin{aligned} \mathrm{DAP} & \geq 10 \mathrm{~cm} \mathrm{y} \\
& <40 \mathrm{~cm}\end{aligned}$ & $\begin{array}{l}\text { A lo largo de } \\
\text { transectos (10 } \\
\text { m a cada lado) }\end{array}$ & 155 & 10 & 0.06 & 0.053 \\
\hline Adultos & $\mathrm{DAP} \geq 40 \mathrm{~cm}$ & $\begin{array}{l}\text { A lo largo de } \\
\text { transectos ( } 50 \\
\text { m a cada lado) }\end{array}$ & 155 & 110 & 0.71 & 0.65 \\
\hline
\end{tabular}

Cuadro 1. Resultado consolidado de las evaluaciones de regeneración natural, plantones, latizales, fustales e individuos adultos. Comparativo con estudio realizado por Romo (2004).

marcadores moleculares han demostrado que la entidad denominada $D$. micrantha "morfotipo 1 " en realidad corresponde a $D$. ferrea (Ducke) Ducke. Por lo tanto, la población de Shihuahuaco evaluada en este trabajo corresponderían a Dipteryx micrantha "morfotipo 1" (= D. ferrea) (Aldana et al. 2016; Garcia-Davila et al. 2019; Honorio et al. 2020). Sin embargo, debido a los pocos estudios taxonómicos o dendrológicos en nuestra área de estudio se ha optado por continuar denominando a la población evaluada bajo el nombre de $D$. micrantha. Esperamos que futuros estudios en la concesión de Arbio-Perú sean enfocados en la caracterización morfológica de los individuos presentes para determinar con certeza a que entidad corresponden.

Por otro lado, y en relación con los resultados obtenidos del conteo de regeneración natural de Shihuahuaco en la concesión de Arbio-Perú, se ha encontrado que la distribución espacial de esta especie no es regular, el $82 \%$ de la RN se ha concentrado asociada con un solo árbol maduro. Además, la casi inexistente presencia de plantones (solo se encontraron 2 individuos) indicaría el bajo nivel de supervivencia de la RN. Adicionalmente, la ausencia de latizales (DAP $\geq 4 \mathrm{~cm} \mathrm{y}<10 \mathrm{~cm}$ ), refuerza el hallazgo en relación con el bajo nivel de supervivencia de la $\mathrm{RN}$ de esta especie.

La densidad de individuos juveniles (DAP $\geq$ $10 \mathrm{~cm} \mathrm{y}<40 \mathrm{~cm}$ ) en este bosque equivale a 0,06 ind/ha, ligeramente mayor al resultado obtenido por Romo (2004) en los bosques de Cocha Cashu (PN Manu), que fue de 0,053 ind/ha. Lo mismo sucedió en el caso de individuos adultos (DAP $\geq 40 \mathrm{~cm}$ ), cuya densidad resultó de $0,71 \mathrm{ind} / \mathrm{ha}$ en comparación con aquellos de Cocha Cashu - PN Manu (0,65 ind/ha). Este contraste en la estructura poblacional de la especie Dipteryx micrantha entre estos dos sitios de Madre de Dios, podría ser resultado de las condiciones ecológicas antes que, por el estado de conservación del bosque, dado que el Parque Nacional del Manu tienen más 47 años desde su establecimiento como área protegida, implementando mecanismos de conservación, en comparación con el área de la concesión Arbio - Perú que inició sus actividades de conservación desde el 2010. 
En base a los resultados de la evaluación de las edades estimadas en este estudio, es evidente que, bajo condiciones de bosques naturales, Dipteryx micrantha es una especie de muy lento crecimiento. Tanto así, que al aplicar la formula alométrica calculada en base a los estudios de Jenkins (2009), a esta especie le tomaría 322 años alcanzar el Diámetro Mínimo de Corta (DAP $51 \mathrm{~cm}$ - en concordancia con lo aprobado mediante Resolución Jefatural $\mathrm{N}^{\circ} 458$-2002-INRENA). En definitiva, el aprovechamiento sostenible de esta población no es posible, dado que no se dan las condiciones para la renovación natural de los individuos adultos. En este sentido, queda pendiente realizar más estudios para determinar las edades de otras especies de árboles de madera dura del bosque amazónico para verificar la sostenibilidad de su aprovechamiento actual.

En condiciones similares, y de acuerdo con los estudios realizados por Chambers et al. (1998) en especies tropicales de madera dura, se confirma que la tasa de crecimiento de los árboles es muy lenta, llegando a tomar doscientos o trescientos años para que un árbol pequeño crezca, o que una plántula sobreviva y llegue a ser un árbol adulto. Por ejemplo, en aplicación a la Política Nacional Forestal y de Fauna Silvestre, aprobada mediante decreto supremo $\mathrm{N}^{\circ}$ 009-2013-MINAGRI, que en su eje de política 2 "Sostenibilidad", la que refuerza la conservación de los bosques inundables a través de prácticas de manejo sostenibles, la reposición de árboles para su cosecha es la base, por lo que cosechar árboles mayores de $51 \mathrm{~cm}$ de DAP (DMC) de D. micrantha no es posible en los ciclos de corta que duran entre 20-30 años que actualmente se utilizan.

El estudio de Jenkins (2009) coincide con las estimaciones alométricas para el cálculo de edades, que para la población de Dipteryx micrantha de la cuenca del Río Piedras tiene una distribución tipo "campana", con una media estimada en de 684,83 años (Std +/- 29,5). Esta distribución de edades no asegura la viabilidad de la especie en la zona (dado que existe muy poca capacidad de reclutamiento de individuos juveniles), lo que mostraría que esta población tuvo un crecimiento poblacional y diamétrico notorio hace 600 años, luego de lo cual, el reclutamiento de adultos se redujo de manera significativa.

\section{Conclusiones}

Bajo las condiciones ecológicas actuales del bosque natural en la cuenca del río Las Piedras, las poblaciones Dipteryx micrantha no son viables a largo plazo, debido a la baja proporción de plantones e inexistencia de latizales, lo que no permite la supervivencia de la regeneración natural. Este hecho dificulta que la población existente tenga reemplazo de individuos en el futuro por falta de reclutamiento.

Con base en las clases diamétricas, y aplicando la fórmula alométrica, se estima que el $68,3 \%$ de la población de individuos adultos de Dipteryx micrantha evaluada en la cuenca del río Las Piedras tendría más de 500 años; y el 15,83\% tendría más de 1000 años. Estos datos ratifican que esta población está conformada por individuos muy antiguos y longevos.

\section{Agradecimientos}

El presente trabajo se ha realizado gracias al financiamiento del Programa de Desarrollo Forestal Sostenible, Inclusivo y Competitivo en la Amazonia Peruana del SERFOR. Especialmente al Ingeniero Leoncio Julio Ugarte Guerra, quién acompañó el proceso de diseño metodológico del proyecto, así como la ejecución de este. Agradecemos el apoyo constante de Rocío y Gianella Espinosa Quiñones y del equipo de campo de Arbio Perú: Ronald Taboada, Emerito Ruiz, Luis Llerena Bermúdez y Consuelo Perdomo. También, la ayuda desinteresada de Mónica Romo y Robin Sears, quienes contribuyeron con información y referencias importantes para este estudio.

\section{Bibliografía}

Aldana, DR; García-Dávila, CR; Hidalgo, CG; Flores, GR; Del Castillo-Torres, D; Reynel, C; Pariente, E; Honorio-Coronado, EN. 2016. Análisis morfométrico de las especies de Dip- 

del río Las Piedras, Madre de Dios (Perú)

teryx en la Amazonía peruana. Folia Amazónica 25(2):101-118.

Cardoso, D; Särkinen, T; Alexander, S; Amorim, AM; Bittrich, V; Celis, M; Daly, DC; Fiaschi, P; Funk, VA; Giacomin, LL; Goldenberg, R; Heiden, G; Iganci, J; Kelloff, CL; Knapp, S; Cavalcante de Lima, H; Machado, AFP; dos Santos, RM; Mello-Silva, R; Michelangeli, FA; Mitchell, J; Moonlight, P; Rodrigues de Moraes, PL; Mori, SA; Sacramento, TN; Pennington, TD; Pirani, JR; Prance, GT; Paganucci de Queiroz, L; Rapini, A; Riina, R; Vargas, CA; Roque, N; Shimizu, G; Sobral, M; Stehmann, JR; Stevens, WD; Taylor, CM; Trovó, M; van den Berg, C; van der Werff, H; Viana, PL; Zartman, CE; Campostrini, R. 2017. Amazon plant diversity revealed by a taxonomically verified species list. Proceedings of the National Academy of Sciences of the United States of America 114(40):1-6. DOI: https://doi.org/10.1073/ pnas. 1706756114 .

Chambers, JQ; Higuchi, N; Schimel, JP. 1998. Ancient trees in Amazon. Nature 391:135-136.

Díaz, R. 2018. Variabilidad genética de "Shihuahuaco" Dipteryx micrantha en poblaciones naturales de la Amazonía peruana, mediante el uso de marcadores microsatélites. Tesis Ing. Ecología de Bosques Tropicales. Iquitos, Perú, UNAP. 81 p.

Garcia Davila, C; Aldana, DR; Renno, JF; Diaz, R; Hidalgo, G; Flores, G; Castro Ruiz, D; Mejía de Loayza, E; Angulo, C; Mader, M; Tysklind, N; Paredes Villanueva, K; Del Castillo, D; Degen, B; Honorio, EN. 2019. Molecular evidence for three genetic species of Dipteryx in the Peruvian Amazon. Genetica: An International Journal of Genetics and Evolution (148):1-11. DOI: $\quad$ https://doi.org/10.1007/s10709-01900082-2.

Hammer, H; Ryan PD. 2001. PAST: Paleontological Statistics Software package for education and data analysis. Paleontología Electrónica 4(1):1-9.

Honorio, EN; Blanc-Jolivet, C; Mader, M; García-Dávila, CR; Aldana, D; Del Castillo, D; Flores, G; Hidalgo, G; Sebbenn, A; Me-
yer-Sand, B; Paredes-Villanueva, K; Tysklind, N; Troispoux, V; Massot, M; Carvalho, C; de Lima, H; Cardoso, D; Degen, B. 2020. SNP Markers as a Successful Molecular Tool for Assessing Species Identity and Geographic Origin of Trees in the Economically Important South American Legume Genus Dipteryx. Journal of Heredity 111(4):346-356. DOI: https://doi.org/ doi:10.1093/jhered/esaa011.

Jenkins, HS. 2009. Amazon Climate Reconstruction Using Growth Rates and Stable Isotopes of Tree Ring Cellulose from the Madre de Dios Basin, Peru. Dissertation for Ph.D. North Carolina, United States of America, Graduate School of Duke University. 239 p.

Laurance, WF; Delamônica, P; Laurance, SG; Vasconcelos, HL; Lovejoy; TE. 2000. Rainforest fragmentation kills big tree. Nature 404:836.

MINAGRI (Ministerio de Agricultura y Riego, Perú). 2013. Metodología del inventario nacional forestal del Perú: diseño y planificación. Lima, Perú, INF. 38 p. Proyecto Inventario Nacional Forestal y Manejo Forestal Sostenible ante el Cambio Climático.

MINAM (Ministerio del Ambiente, Perú). 2019. Mapa nacional de ecosistemas del Perú. Lima, Perú. 63 p.

Oliveira, PJ; Asner, GP; Knapp, DE; Almeyda, A; Galván-Gildemeister, R; Keene, S; Raybin, RF; Smith, RC. 2007. Land-Use Allocation Protects the Peruvian Amazon. Science 317(5842):1233-1236. DOI: https://doi. org/10.1126/science.1146324.

OSINFOR (Organismo de Supervisión de los Recursos Forestales y de Fauna Silvestre, Perú). 2013. Distribución de las especies forestales del Perú. Lima, Perú. 294 p.

Putzel, L. 2010. The tree that held up the forest: Shihuahuaco (Dipteryx spp.) and the Chinese timber trade. New York, United States of America, City University of New York. 288 p.

Putzel, L; Padoch, C; Pinedo-Vasquez, M. 2008. The Chinese Timber Trade and the Logging of Peruvian Amazonia. Conservation biology: the journal of the Society for Conservation Biology 
22(6):1659-61. DOI: https://doi.org/10.1111/ j.1523-1739.2008.01065.x.

Resolución de Dirección Ejecutiva $\mathrm{n}^{\circ} 118$ 2019-MINAGRI-SERFOR-DE. Lista oficial de especies forestales. Diario Oficial El Peruano. Perú. 27 may. 2019.

Romo, M. 2004. Regeneration ecology and population structure of the emergent tree Dipteryx micrantha (Fabaceae) in floodplain forests of the Manu river, Amazonian Peru. Turku, Finland, s.e. (Serie Annales Universitatis Turkuensis. Sarja-Ser. A11-Tom 180).

Romo, M; Roukolainen, K; Rajaniemi, S. 2004. Population structure and recruitment of an emergent tree, Dipteryx micrantha, in different habitats of a Peruvian floodplain forest. Ann. Univ. Turku. Sarja-Ser. AII IV, 1-19.

SENHAMI (Servicio Nacional de Meteorología e Hidrología del Perú). 2020. Mapa climático. (en línea, sitio web). Consultado 20 jul. 2020. Disponible en https://www.senamhi. gob.pe/?p=pronostico-detalle-turistico\&localidad $=0027$. 